\title{
Inositol pyrophosphates promote tumor growth and metastasis by antagonizing liver kinase B1
}

\author{
Feng Rao ${ }^{a, 1}$, Jing Xu ${ }^{a, 1}$, Chenglai Fu ${ }^{a, 1}$, Jiyoung Y. Cha ${ }^{a}$, Moataz M. Gadalla ${ }^{a, b}$, Risheng Xu ${ }^{a, b}$, James C. Barrow ${ }^{b, c}$, \\ and Solomon H. Snyder ${ }^{a, b, d, 2}$
}

\begin{abstract}
Contributed by Solomon H. Snyder, December 25, 2014 (sent for review November 8, 2014)
The inositol pyrophosphates, molecular messengers containing an energetic pyrophosphate bond, impact a wide range of biologic processes. They are generated primarily by a family of three inositol hexakisphosphate kinases (IP6Ks), the principal product of which is diphosphoinositol pentakisphosphate (IP7). We report that IP6K2, via IP7 synthesis, is a major mediator of cancer cell migration and tumor metastasis in cell culture and in intact mice. IP6K2 acts by enhancing cell-matrix adhesion and decreasing cell-cell adhesion. This action is mediated by IP7-elicited nuclear sequestration and inactivation of the tumor suppressor liver kinase B1 (LKB1). ACcordingly, inhibitors of IP6K2 offer promise in cancer therapy.
\end{abstract}

${ }^{a}$ The Solomon H. Snyder Department of Neuroscience, ${ }^{b}$ Department of Pharmacology and Molecular Sciences, ${ }^{\mathrm{C}}$ Lieber Institute for Brain Development, and ${ }^{\mathrm{d}}$ Department of Psychiatry and Behavioral Sciences, The Johns Hopkins University School of Medicine, Baltimore, MD 21205

IP6K | cell-cell adhesion | cell-matrix adhesion | metastasis | LKB1

nositol pyrophosphates, conserved eukaryotic messenger molecules with a pyrophosphate bond, mediate numerous physiologic processes including regulation of Akt (1), insulin secretion (2), ATP production (3), DNA repair (4), and damage response (5). Exemplified by diphosphoinositol pentakisphosphate (PPIP5, IP7), inositol pyrophosphates are primarily generated by a family of three inositol hexakisphosphate (IP6) kinases (IP6K) $(6,7)$ and also may be formed by a more recently described group of IP6/7 kinases (8). IP6K1 and IP6K2 are widely distributed, whereas IP6K3 is expressed primarily in the brain (9). A related enzyme, inositol polyphosphate multikinase (IPMK), converts IP3 to IP4 and IP5, displays physiologic PI3 kinase activity (10), and noncatalytically acts as a transcriptional coactivator (11) and stabilizer of the mTOR complex-1 (12). Whether IP6Ks and IPMK play direct roles in tumor progression remains unexplored.

Tumor cell metastasis requires loss of cell-cell adhesion and gain of migratory and invasive properties, a collective program known as epithelial-mesenchymal transition (EMT), which is also critical for embryonic development (13). Cell-cell adhesion and cell migration/invasion are primarily driven by E-cadherinmediated cell clustering (14) and focal-adhesion-based cellmatrix interactions (15), respectively. Whether these two distinct adhesion processes are concurrently or independently regulated remains poorly understood.

The tumor suppressor LKB1 has been separately reported to inhibit FAK activation (16) and to enhance E-cadherin expression (17-19). LKB1 is mutated in Peutz-Jeghers syndrome patients who tend to develop cancer at multiple sites (20). As a master kinase controlling the activity of AMPK and 13 other AMPK-like kinases (21), LKB1 mediates diverse cellular processes such as polarity, adhesion, metabolism, tumor growth/metastasis, and neuronal axon initiation/branching (22). Unlike conventional protein kinases, LKB1 is not activated by activation loop phosphorylation but by protein-protein interaction with two other subunits of the heterotrimeric holoenzyme: STRAD and Mo25. LKB1 can also exist as an inactive nuclear monomer. STRAD binds and stabilizes LKB1 (23) in the cytosol, where it is phosphorylated at serine 428 by PKC $\zeta$ (24). Whether cytosolic localization enhances S428 phosphorylation, or vice versa, remains to be established. Thus, mechanisms determining LKB1's localization and activity remain unclear. It is also not clear whether LKB1's localization is regulated by additional signaling components.

We now report that IP6K2, via its catalytic activity, promotes cancer cell migration, invasion, and tumor metastasis by upregulating cell-matrix adhesion and downregulating cell-cell adhesion. Mechanistically, IP6K2/IP7 promotes nuclear sequestration of the tumor suppressor LKB1. This action of IP7 represents a novel mode of LKB1 regulation and also makes IP6K2 a plausible anticancer target.

\section{Results}

IP6K2 Up-Regulates Focal Adhesion and Down-Regulates Cell-Cell Adhesion. While subculturing the colorectal cancer cell line HCT116, we noted that cells with IP6K2 deletion spread more slowly than wild-type cells (Fig. $1 A$ and $B$ ) and display attenuated cell-matrix adhesion (Fig. $1 C$ ). Cell-matrix adhesion and spreading are driven by the formation of focal adhesions (15). In fibronectincoated plates we confirm that focal adhesion, assessed by staining of the focal adhesion protein vinculin (Fig. 1D), or levels of phosphorylated focal adhesion kinase (p-FAK) (Fig. 1E) are greatly reduced in IP6K2 knockout cells. During routine passage, focal adhesions and p-FAK levels are also markedly diminished in IP6K2 knockout cells $22 \mathrm{~h}$ after seeding (Fig. S1 $A$ and $B$ ). After $44 \mathrm{~h}$ in culture, spreading (Fig. $1 A$ ) and p-FAK levels (Fig. S1B) are no longer diminished in IP6K2 deleted cells. However, the knockout cells cluster more tightly (Fig. $1 A$ ), a phenomenon that is more pronounced in polyHEMA plates wherein cells are cultured in suspension (Fig. $1 F$ ). The increased clustering implies greater cell-cell adhesion in the IP6K2 deleted cells, which is supported by substantially increased immunohistochemical staining and Western blotting for the adherens junction protein E-cadherin (Fig. $1 G$ and $H$ ), a major mediator of cell-cell adhesion (14).

\section{Significance}

Inositol pyrophosphates are messenger molecules incorporating the energetic pyrophosphate bond. Although they have been implicated in diverse biologic processes, their physiologic functions remain enigmatic. We show that the catalytic activity of inositol hexakisphosphate kinase 2 (IP6K2), one of the principal enzymes generating the inositol pyrophosphate IP7 (5-diphosphoinositolpentakisphosphate), mediates cancer cell migration and tumor metastasis both in cell culture and intact mice. In this process, IP6K2 diminishes cell-cell adhesion, enabling cells to invade the intercellular matrix. Drugs that inhibit IP6K2 may be beneficial in cancer therapy.

Author contributions: F.R., J.X., and S.H.S. designed research; F.R., J.X., C.F., J.Y.C., M.M.G. and R.X. performed research; J.C.B. contributed new reagents/analytic tools; F.R., J.X., and C.F. analyzed data; and F.R. and S.H.S. wrote the paper.

The authors declare no conflict of interest.

${ }^{1}$ F.R., J.X., and C.F. contributed equally to this work.

${ }^{2}$ To whom correspondence should be addressed. Email: ssnyder@jhmi.edu.

This article contains supporting information online at www.pnas.org/lookup/suppl/doi:10. 1073/pnas.1424642112/-/DCSupplemental. 




$\mathrm{E}$

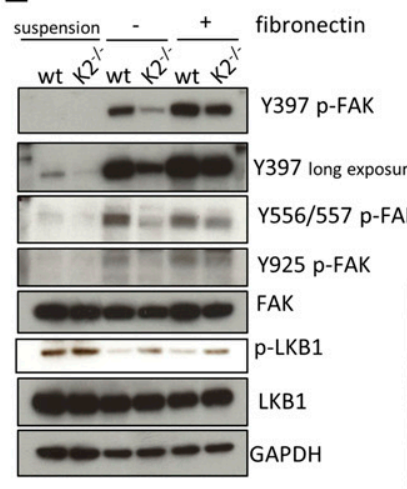

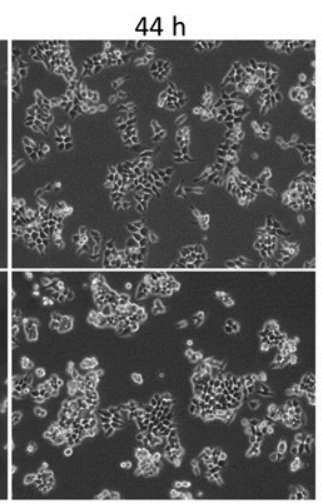



$\mathrm{F}$
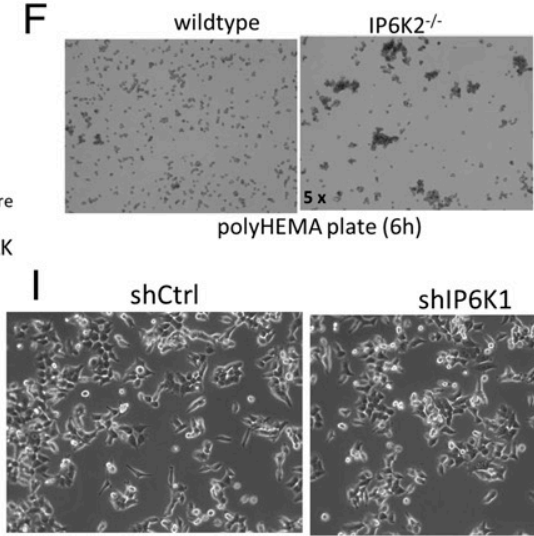



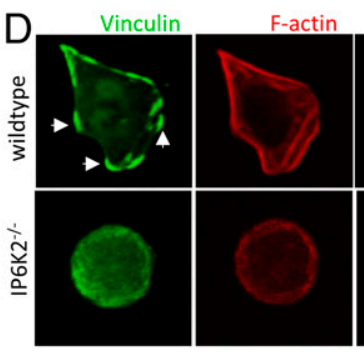

E-cadherin
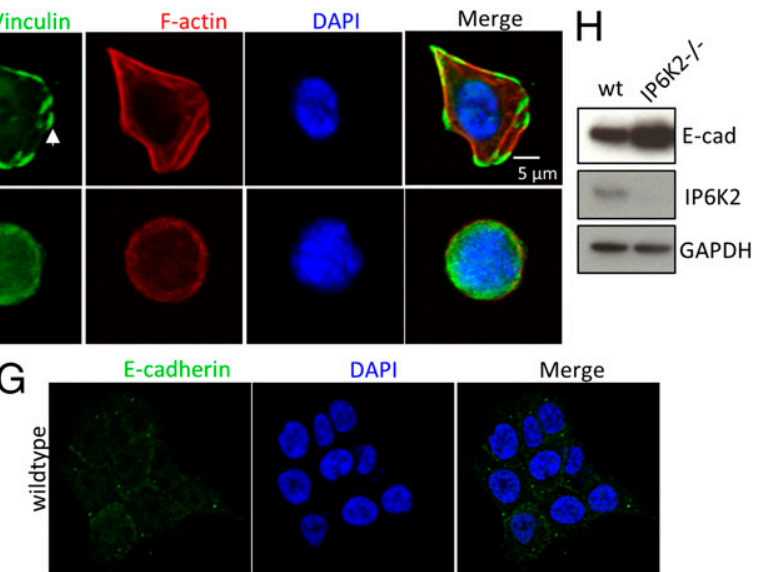

DAPI
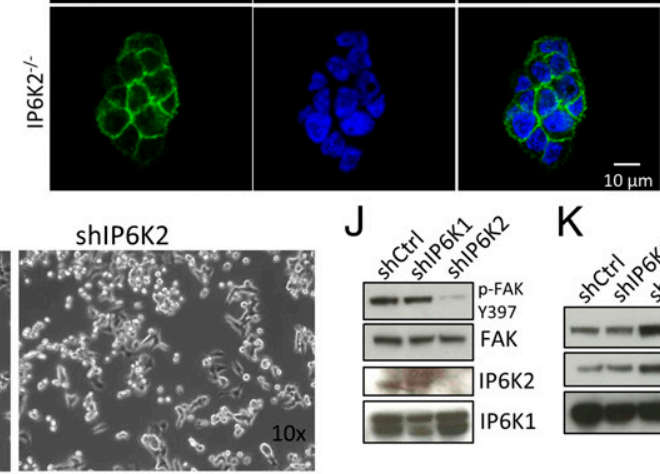

$\mathrm{J}$



$\mathrm{K}$

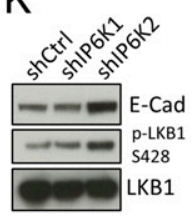

Fig. 1. IP6K2 up-regulates cell-matrix adhesion and down-regulates cell-cell adhesion. (A) Morphology of wild-type and IP6K2 ${ }^{-/-} \mathrm{HCT} 116$ cells 22 and $44 \mathrm{~h}$ after seeding. (B) Percentage of cells spread at $22 \mathrm{~h}$. (C) Cell-matrix adhesion force measured by the centrifugation assay $22 \mathrm{~h}$ after plating. ( $D$ ) Immunostaining of vinculin and actin in wild-type and knockout cells. Cells were starved overnight, trypsinized, and then plated on gelatin (10 $\mu \mathrm{g} / \mathrm{mL}$ )-coated plates for $1 \mathrm{~h}$ before staining. Arrowheads point to focal adhesions. $(E)$ Levels of phospho-FAK and phospho-LKB1 determined by Western blot. Cells were in suspension on regular plates or on fibronectin-coated plates for 30 min before harvest. $(F)$ Morphology of wild-type and IP6K2 ${ }^{-l-}$ HCT116 cells grown in suspension. (G) Immunostaining of E-cadherin in wild-type and knockout cells $44 \mathrm{~h}$ after plating. $(H)$ Levels of E-cadherin determined by Western blot $44 \mathrm{~h}$ after plating. $(I)$ Morphology of wild-type or IP6K1, IP6K2 knockdown HCT116 cells $28 \mathrm{~h}$ after seeding. ( $)$ Levels of phospho-FAK in IP6K1 or IP6K2 knockdown HCT116 cells, determined by Western blot. $(K)$ Levels of E-cadherin and phospho-LKB1 in IP6K1 or IP6K2 knockdown HCT116 cells. Error bars represent means \pm SD; $n=3$ in triplicate $(* * P<0.01$, Student's $t$ test).

We validated the data from knockout cell lines by using shRNA knockdown, wherein knockdown of IP6K2 but not IP6K1 in HCT116 cells delays cell spreading, decreases FAK phosphorylation, and increases E-cadherin levels (Fig. $1 I-K$ ), suggesting selective effects by IP6K2, which is in line with our previous finding that IP6K2 is primarily responsible for IP7 generation in this cell line (25). Moreover, the importance of IP6K2 for cell adhesion/spreading is general. Thus, knockdown of IP6K2 in the breast cancer cell line MCF7 or the lung cancer cell line H1299 reduces IP7 levels, delays cell spreading, and augments cell clustering (Fig. S1 $C-J$ ). These phenotypes are substantiated by analysis of molecular markers, as IP6K2 deletion elicits decreased FAK phosphorylation and increased levels of E-cadherin in MCF7 cells (Fig. S1 $K$ and $L$ ). In $\mathrm{H} 1299$ cells E-cadherin is not detectable (18), but the tight junction protein zona occludin-1, another mediator of cell-cell adhesion, is increased with IP6K2 deletion (Fig. S1M). Given that HCT116, MCF7, and H1299 cells are of different tissue origin and exhibit vastly different E-cadherin levels as well as spreading/adhesion speed (Fig. 1 and Fig. S1), our data suggest that IP6K2 has a widespread role in promoting cell-matrix interactions while suppressing cell-cell adhesion.

IP6K2 Mediates Cell Migration/Invasion and Tumor Growth/Metastasis. The diminished cell-matrix adhesion and augmented cell-cell adhesion with IP6K2 deletion suggest that IP6K2 mediates EMT properties such as cancer cell migration and invasion and might therefore be anticipated to impact tumor growth and/or metastasis.
To examine this possibility, we monitored HCT116 cell growth, which is delayed in IP6K2 knockout preparations (Fig. 2A), consistent with delayed cell adhesion and spreading in culture plates (Fig. 1). In the same cells, anchorage-independent growth, reflecting cell transformation and measured by the soft agar colony formation assay, is diminished seven- to eightfold (Fig. 2B), suggesting a loss of malignant transformation associated with IP6K2 deletion. Cell migration and invasion, EMT properties measured by the Transwell migration and Matrigel invasion assays, respectively, are reduced by $70-80 \%$ (Fig. $2 C$ ) in the absence of IP6K2. Cell migration, assessed by the scratch-wound healing assay, also declines significantly in IP6K2-depleted MCF7 and H1299 cells (Fig. S2 $A-C$ ). In vitro tumor invasion, monitored by tumor sphere growth in a collagen invasion assay, is greatly reduced with IP6K2 deletion (Fig. 2D). Taken together, these in vitro data suggest that IP6K2 is required for cancer cell aggressiveness.

We then explored the influence of IP6K2 deletion on tumors in intact animals (Fig. $2 E-G$ ). Subcutaneous xenograft tumors grown from IP6K2 knockout cells are reduced in size (Fig. 2E), consistent with a role for IP6K2 in tumor growth. To study the influence of IP6K2 on tumor metastasis, we used an established orthotopic model wherein small pieces of xenograft tumors are implanted into the cecum of nude mice (26) and liver metastases are monitored (Fig. $2 F$ and $G$ ). The size of orthotopically transplanted tumors (Fig. $2 F$ ) and the percentage of metastases to liver (Fig. 2G), the most common site of metastasis for 


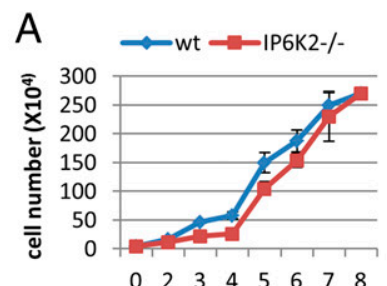

$\begin{array}{llll}0 & 2 & 3 & 4 \\ & & \\ & & \end{array}$

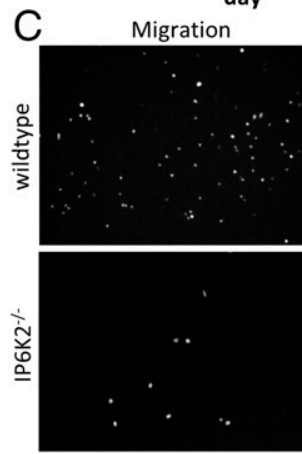

transwell migration

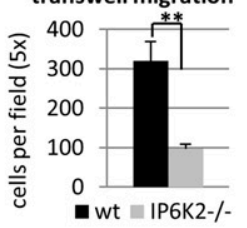

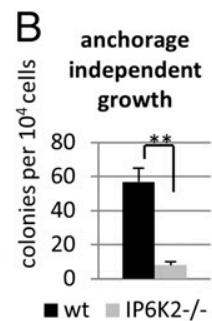

nvasion

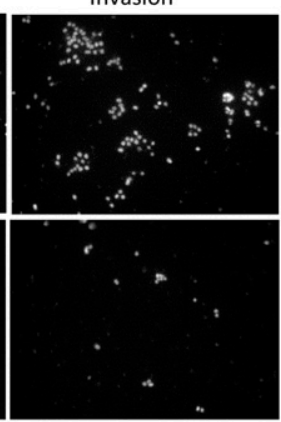

Matrigel Invasion



D
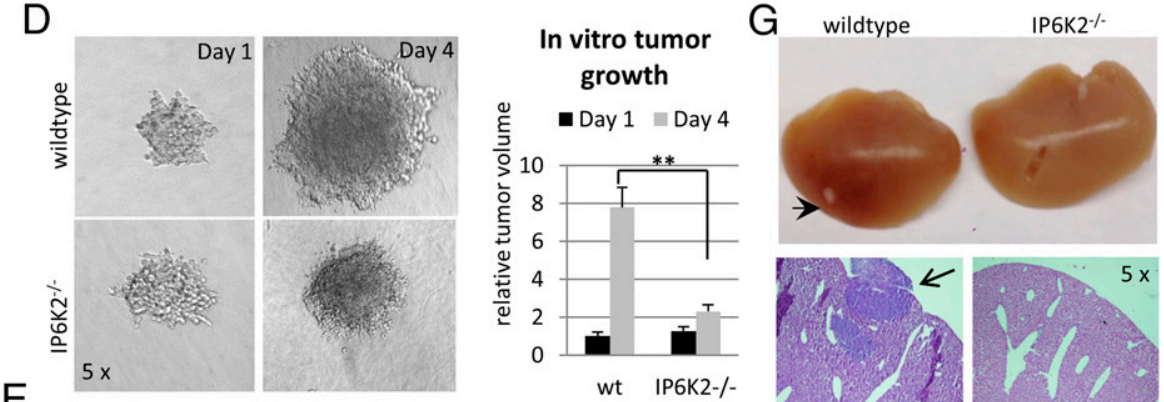

E
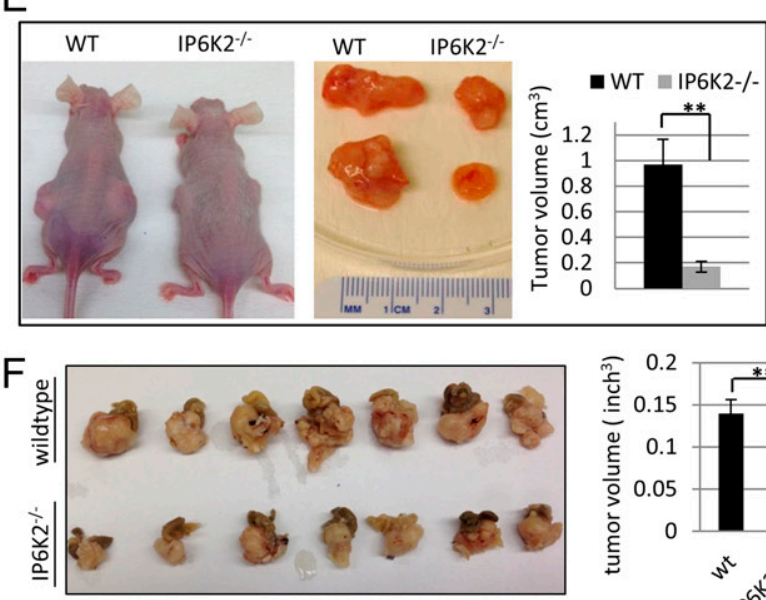

Fig. 2. IP6K2 mediates cell migration, invasion, tumor cell growth, and metastasis. ( $A$ ) Growth curve of wild-type and IP6K2 null cells. ( $B$ ) Anchorage-independent growth in agarose. (C) Cell migration (Left) through transwell and invasion through Matrigel (Right). Cells that have migrated/invaded were stained with DAPI and imaged using a fluorescence microscope. $(D)$ In vitro tumor sphere growth. $(E)$ Subcutaneous xenograft from wild-type and IP6K2 ${ }^{-/-}$ HCT116 cells. Implanted tumors were grown for 3 wk. $(F)$ Tumor volume of orthotopic transplant from wild-type and IP6K2 ${ }^{-/-}$HCT116 cells. (G) Liver metastasis from orthotopic transplant. The H\&E staining reveals metastatic spots. Error bars represent means \pm SD; $n=3$ in triplicate $(* \star P<0.01$ and $* P<0.05$, Student's $t$ test).

HCT116 cells (26), are significantly less in mice implanted with tumors arising from IP6K2 knockout cells. Thus, IP6K2 mediates tumor growth and metastasis in vivo.

IP7 Synthesis Is Required for the Protumorigenic Actions of IP6K2. The inositol polyphosphate kinases, including IP6Ks, have both catalytic activity-dependent and -independent functions $(1,5,11$, $27,28)$. To examine whether the oncogenic actions of IP6K2 depend on its catalytic activity, we established rescue cell lines by retroviral expression of wild-type (WT-R) or kinase-dead K222A IP6K2 (29) (KD-R) in IP6K2 ${ }^{-7-}$ cells. Compared with the vector control-rescued cell line, WT-R, but not KD-R, markedly augments cell adhesion, spreading, anchorage-independent growth, Transwell migration, Matrigel invasion, and in vitro tumor invasion (Fig. $3 A$ and $B$ and Fig. S3 $A-C$ ) while repressing cell clustering (Fig. $3 A$ ). Consistent with the cellular data, overexpressing wildtype but not kinase-dead IP6K2 also rescues the abnormalities in FAK phosphorylation and E-cadherin levels (Fig. $3 C$ and $D$ ). Thus, the ability to generate IP7 is critical for IP6K2 to up-regulate focal adhesion and down-regulate cell-cell adhesion.

We further examined the influence of IP6K2 catalytic activity on tumorigenesis in intact animals. In nude mice, xenograft tumors (Fig. 3E) grown from WT-R cells are larger than those elicited by KD-R cells or controls. In the orthotopic model, mice transplanted with tumors arising from WT-R cells are less active and become moribund sooner. The extent of metastases in liver and s.c. tissues, as well as the number and size of intestinal polyps, is substantially greater with tumors arising from WT-R cells than KD-R cells (Fig. $3 F-I$ and Fig. S3D). Fecal blood is substantial in WT-R but not KD-R mice (Fig. S3 $E$ and $F$ ), resembling mice with mutations in the colorectal cancer tumor suppressor adenomatous polyposis coli (30). Together, these data suggest that IP7 synthesis is essential for IP6K2 to impact tumor progression and metastasis.

IP6K2/IP7 Inactivates LKB1 by Promoting Its Nuclear Localization. What mechanisms might account for the tumorigenic influences of IP6K2? Bioinformatic analysis using the Predicting ProteinProtein Interactions platform (31) indicates genetic interactions of IP6K2, but not IP6K1 or IP6K3, with STRAD $\beta$, a subunit of the tumor suppressor LKB1 (Fig. S4A) (20, 23). IPMK, an upstream kinase in the inositol polyphosphate synthesis pathway, influences LKB1 activity in a catalytic activity-dependent manner (32), suggesting that higher inositol phosphates regulate LKB1. LKB1 has been reported to suppress FAK activity-dependent migration/invasion and metastasis $(16,33,34)$, as well as to enhance E-cadherin levels $(17,19)$. Using MCF7 and H1299 cells with LKB1 depletion, we confirm that LKB1 down-regulates cell spreading (Fig. S1 $D$ and $H$ ) and p-FAK levels (Fig. S1K), up-regulates cellcell adhesion (Fig. S1 $E-G, I$, and $J$ ) and E-cadherin/zonaoccludin-1 levels (Fig. S1 $L$ and $M$ ), and inhibits cell migration (Fig. S2 $A-C$ ). These findings indicate that LKB1 concurrently regulates both cell-cell and cell-matrix adhesion.

We then examined whether IP6K2 influences cell-matrix adhesion and cell-cell adhesion by suppressing LKB1. In line with this notion, knockdown of LKB1 reverses defects in FAK phosphorylation (Fig. 4A) and cell morphology (Fig. S4B) in IP6K2 null cells. LKB1 activates various tyrosine phosphatases (35), which might underlie its negative regulation of p-FAK $(16,33)$. Notably, tyrosine phosphatase activity is elevated in IP6K2 null cells (Fig. 
A



B



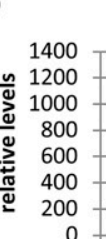

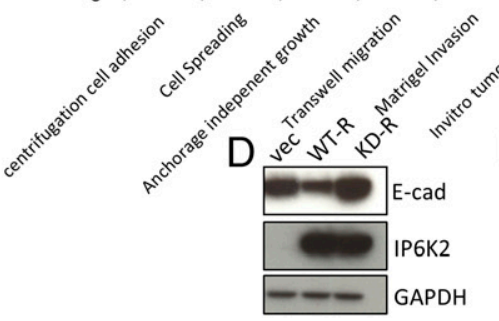

E

$\square \mathrm{Vec} \backsim \mathrm{WT}-\mathrm{R} \backsim \mathrm{KD}-\mathrm{R}$

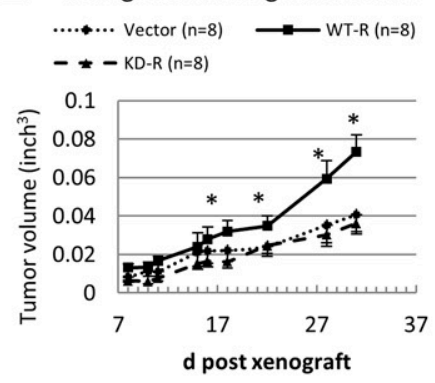

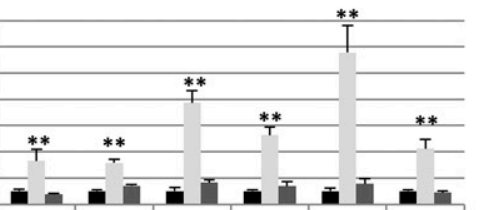

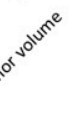

F $\quad$ liver met

n polyps

m bloody stool


Fig. 3. Catalytic activity of IP6K2 is required for its actions. $(A)$ Wild-type but not catalytically dead (K222A) IP6K2 rescues the defect in cell spreading ( $i$ ), cellcell adhesion (ii), cell clustering (iii), focal adhesion formation (iv), and E-cadherin staining (v). (B) Wild-type but not catalytically dead (K222A) IP6K2 rescues the defect in cell adhesion force, anchorage-independent growth, transwell migration, Matrigel invasion, and in vitro tumor sphere formation. Data are normalized to values from mock-rescued (vector-only) cells. $(C)$ Levels of phospho-FAK and phospho-LKB1 in the three rescued cell lines with/without fibronectin treatment. $(D)$ Levels of E-cadherin in the three rescued cell lines. $(E)$ Growth curve of xenograft tumors from the three rescued cell lines. $(F)$ Percentage of orthotopic-transplanted mice with liver metastasis, s.c. metastasis, intestinal polyps, and bloody stool. (G) Typical image of orthotopic-transplanted mice. The one transplanted using wild-type IP6K2-rescued cells displays s.c. tumor metastasis. ( $H$ ) Mice orthotopically transplanted using wild-type IP6K2-rescued cells display intestinal polyps. (I) Mice orthotopically transplanted with wild-type IP6K2-rescued cells have a higher percentage of liver metastasis and more metastatic spots. Error bars represent means \pm SD; $n=3$ in triplicate $(* * P<0.01$ and $* P<0.05$, Student's $t$ test).

$4 B$ ), the low p-FAK level of which is rescued by a tyrosine phosphatase inhibitor (Fig. $4 C$ ), consistent with the notion that IP6K2 suppresses LKB1 to inactivate tyrosine phosphatases, thereby promoting p-FAK levels and focal adhesion. Finally, IP6K2 knockdown in the A549 lung carcinoma cell line, which is deficient in LKB1 (36), has little effect on p-FAK and E-cadherin levels (Fig. S4C) or cell morphology (Fig. S4D). Together, these data from cells with LKB1 knockdown or deletion indicate that IP6K2 acts via LKB1.

How does IP6K2 antagonize LKB1's tumor-suppressive actions? LKB1's kinase activity is determined by its intracellular distribution, as its substrates are predominantly cytosolic (20). LKB1 appears largely cytosolic in IP6K2 null HCT16 cells, but mostly nuclear in wild-type cells (Fig. 4D). Coexpression of wild type but not kinase-dead IP6K2 increases its nuclear localization (Fig. 4E), suggesting that IP6K2 represses LKB1 function by promoting its nuclear import via IP7.

LKB1 phosphorylation at serine 428 by $\mathrm{PKC} \zeta$ is associated with cytosolic localization, whereas unphosphorylated LKB1 is largely nuclear (24), although causal links of S428 phosphorylation status and LKB1 localization remain unclear. In multiple cell lines wherein IP6K2 deletion or knockdown leads to diminished p-FAK and augmented E-cadherin levels, LKB1 phosphorylation at $\mathrm{S} 428$ is invariably increased (Fig. $1 E$ and $K$; Fig. S1 $B, L$, and $M$ ). Overexpressing wild-type but not kinasedead IP6K2 decreases phosphorylation of endogenous as well as coexpressed LKB1 in HCT116 and HEK293 cells (Figs. 3C and $4 F$; Fig. S4E) without apparent effect on the upstream kinase PKCל (Fig. S4F). These observations imply that IP6K2 regulates
LKB1 S428 phosphorylation and localization at the level of LKB1 itself, which is consistent with the reversible coimmunopreciptation, and hence complex formation, between IP6K2 and LKB1 (Fig. 4F). The catalytic-dead K222A mutation does not influence IP6K2 binding to LKB1, but abolishes the effect on LKB1 S428 phosphorylation, suggesting that local IP7 production is required to inhibit LKB1 phosphorylation and enhance its nuclear localization.

\section{Discussion}

A role for inositol pyrophosphates in cancer has not been hitherto reported. Here we have established that IP6K2 binds to LKB1, with the IP7 generated by IP6K2 eliciting nuclear sequestration of LKB1. IP6K2 thereby promotes cell invasion and metastasis via LKB1 inactivation. IP6K2 deletion diminishes these processes in cellular preparations and in intact animals, effects rescued by re-expression of wild-type IP6K2 but not the catalytically incompetent K222A mutant. These findings imply that IP6K2-specific inhibitors will exert antitumor actions.

Recent evidence suggests that the antimetastatic actions of LKB1 could stem from AMPK-independent down-regulation of the cell-matrix focal adhesion process $(16,33,34)$ or up-regulation of E-cadherin-based cell-cell adhesive properties $(17,19)$. Here we show that these two opposing processes can be simultaneously altered by LKB1 knockdown, suggesting that LKB1 controls the balance between cell-cell and cell-matrix adhesion. Furthermore, inactivation of LKB1 by IP6K2/IP7 shifts this balance 
A

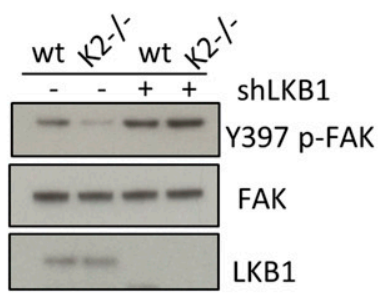

D



B

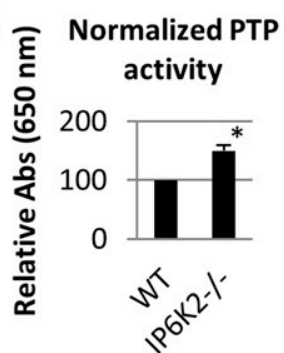

C

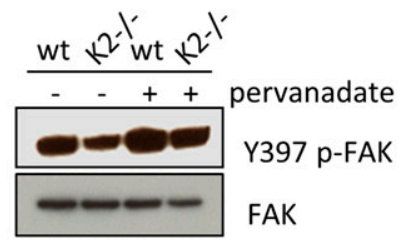

DAPI
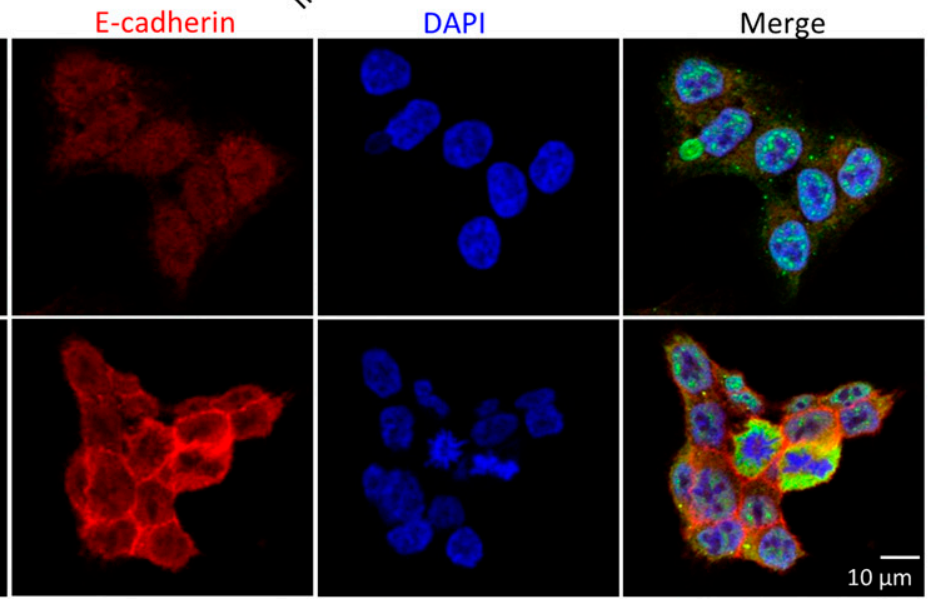

E

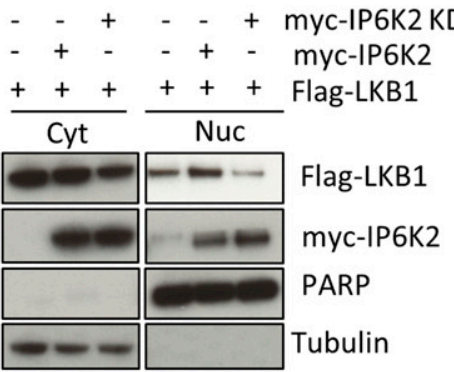

$\mathrm{F}$

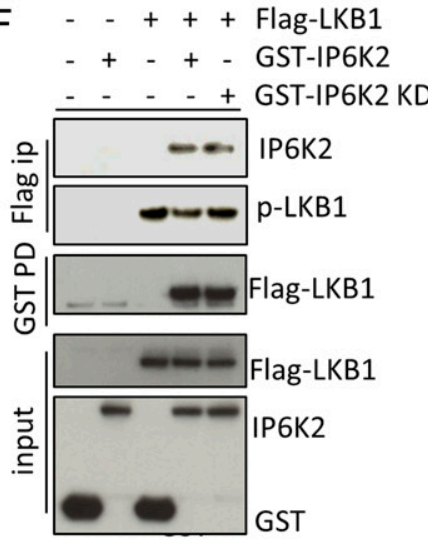

Fig. 4. IP7 promotes nuclear localization of LKB1 by disrupting LKB1-HSP90 binding. (A) LKB1 knockdown rescues the defect in FAK phosphorylation in IP6K2 null cells. (B) Enhanced protein tyrosine phosphatase activity in IP6K2 null cells. (C) The general tyrosine phosphatase inhibitor pervanadate (50 $\mu$ M, 30 min) augments FAK phosphorylation in IP6K2 null cells. (D) Immunostaining of LKB1 reveals IP6K2-dependent differential localization. (E) IP6K2 coexpresssion enhances nuclear localization of LKB1. Cyt, cytoplasmic; Nuc, nuclear. $(F)$ Coimmunoprecipitation between Flag-LKB1 and GST-IP6K2 wild-type or kinase-dead mutant.

to favor focal adhesion while weakening cell-cell adhesion, leading to cell migration/invasiveness.

IP6K2 was previously reported to mediate apoptosis (37), thereby facilitating the DNA-damage-induced p53 cell-death pathway, and so might be regarded as a potential tumor suppressor (5). On the other hand, IP6K2 is a downstream target of the Wnt/ $\beta$-catenin signaling pathway and participates in its oncogenic activities (38). Moreover, IP6K2 is down-regulated by transcription factors that promote mesenchymal-epithelial transition (39) and by tumor suppressors that inhibit EMT (40). This suggests that IP6K2 promotes mesenchymal processes, consistent with a role in tumor metastasis. Accordingly, IP6K2 could act as a potential tumor suppressor in the initiation stage but contribute to tumor progression and metastasis at later stages. Similar dual roles have been observed for TGF- $\beta 1$, a well-known EMT inducer that is also required for DNA-damaged-induced $\mathrm{p} 53$ activation (41, 42).

Although most kinases are activated through phosphorylation on the activation loop, LKB1 is constitutively active biochemically, but requires cytosolic localization for substrate access (20). Our finding that IP6K2/IP7 promotes LKB1 nuclear sequestration suggests that IP7 antagonizes a cellular factor that holds LKB1 in the cytosol, rendering LKB1 accessible to S428 kinases. Alternatively, IP7 might repress LKB1 phosphorylation by upstream kinase(s), thereby promoting LKB1's nuclear localization. In either case, the IP6K2/IP7 system represents a previously unidentified means of controlling LKB1, potentially enabling dynamic LKB1 activation. Another constitutive kinase, CK2, is also regulated by IP6K2/IP7 (5), suggesting an important role for IP7 in fine-tuning the activity of "constitutive" kinases.

\section{Materials and Methods}

Reagents. Chemicals were from Sigma [sodium orthovanadate (pervanadate), poly-HEMA plates, puromycin], BD Bioscience (Matrigel invasion chambers, control cell culture inserts, and basement membrane matrix, calcein AM fluorescent dye), Fisher Scientific (Caliper Digital), Life Technologies (calcein, AM), and Cytoskeleton Inc. [phalloidin (rhodamine)]. IP6K2, IP6K1, and LKB1 knockdown shRNA constructs were from Sigma. Vectors were from Cell Biolabs (pMXs), Calbiochem (pET26), and GE Healthcare (pGEX6P2). The protein tyrosine phosphatase assay kit was purchased from Millipore and used as described (35). The cell fractionation kit used was from Enzo Life Sciences.

Plasmids. Plasmid pcDNA-FLAG-LKB1 was from Addgene. The pCMV-GSTIP6K2 vector used is as described (5). IP6K2, wild-type, or the K222A mutant were cloned into $\mathrm{pMXs}$ vector at BamHI/Xhol restriction sites and into $\mathrm{PCMV}$ GST vector at Sall/Notl restriction sites. All point mutants were made using a site-directed mutagenesis kit (Stratagene).

Immunoblotting and Immunoprecipitation. Standard methods for cell lysis, immunoprecipitation/GST-pulldown, SDS/PAGE, and Western blot were as described (25).

Cell Culture and Transfection Conditions. HEK293, 293T, 293GP, and HCT116 cells were cultured and transfected as described (25). MEF, MCF7, H1299, and A549 cells were cultured in DMEM/10\% (vol/vol) FBS at $5 \%$ ( $\mathrm{vol} / \mathrm{vol}) \mathrm{CO}_{2}$. Transient transfections were carried out using Polyfect (Qiagen). Lentiviral knockdown cell lines were generated as described (5). IP6K2 null HCT116 cells rescued with pMXS-IP6K2 (wild type or K22A) were generated using retrovirus prepared from a 293GP cell line and maintained in DMEM with $5 \mu \mathrm{g} / \mathrm{mL}$ puromycin.

Cell-Spreading Migration, Invasion, and Anchorage-Independent Growth. Cell spreading at the indicated time points was quantified based on visual examination of light microscopy images. In vitro cell migration and invasion assays were performed as described (43). Anchorage-independent growth was assessed as described (10).

Scratch-Wound Healing Assay. The scratch was made on a confluent monolayer using a yellow pipette tip. Wound closure was then monitored by light microscopy at indicated time points. 
Fluorescence Microscopy. For immunofluorescence microscopy, cells, fixed in $4 \%$ (vol/vol) paraformaldehyde for $15 \mathrm{~min}$, were permeabilized in PBST $(0.3 \%$ Triton X-100). Nonspecific binding was blocked in PBST $(0.1 \%$ Triton X-100) with $10 \%$ goat serum. Antibodies against E-cadherin (1:100), vinculin (1:200), LKB1 (1:200), and phalloidin (rhodamine) were then applied in blocking solution and incubated at $4{ }^{\circ} \mathrm{C}$ overnight. Secondary antibodies conjugated to Alexa-fluor-488/568 were added to the coverslip for $1 \mathrm{~h}$ at room temperature. The coverslips were mounted onto slides using prolong gold (with DAPI) and imaged as previously described (5).

Mice Xenograft and Orthotopic Model. Athymic NCr-nu/nu nude mice from the National Cancer Institute were housed under specific-pathogen-free conditions. Experimental protocols were approved by The Johns Hopkins University Animal Care and Use Committee. The xenograft and orthotopic models were performed as described (26).

1. Chakraborty A, et al. (2010) Inositol pyrophosphates inhibit Akt signaling, thereby regulating insulin sensitivity and weight gain. Cell 143(6):897-910.

2. Illies C, et al. (2007) Requirement of inositol pyrophosphates for full exocytotic capacity in pancreatic beta cells. Science 318(5854):1299-1302.

3. Szijgyarto Z, Garedew A, Azevedo C, Saiardi A (2011) Influence of inositol pyrophosphates on cellular energy dynamics. Science 334(6057):802-805.

4. Jadav RS, Chanduri MV, Sengupta S, Bhandari R (2013) Inositol pyrophosphate synthesis by inositol hexakisphosphate kinase 1 is required for homologous recombination repair. J Biol Chem 288(5):3312-3321.

5. Rao F, et al. (2014) Inositol pyrophosphates mediate the DNA-PK/ATM-p53 cell death pathway by regulating CK2 phosphorylation of Tti1/Tel2. Mol Cell 54(1):119-132.

6. Saiardi A, Erdjument-Bromage H, Snowman AM, Tempst P, Snyder SH (1999) Synthesis of diphosphoinositol pentakisphosphate by a newly identified family of higher inositol polyphosphate kinases. Curr Biol 9(22):1323-1326.

7. Chakraborty A, Kim S, Snyder SH (2011) Inositol pyrophosphates as mammalian cell signals. Sci Signal 4(188):re1.

8. Mulugu S, et al. (2007) A conserved family of enzymes that phosphorylate inosito hexakisphosphate. Science 316(5821):106-109.

9. Saiardi A, Nagata E, Luo HR, Snowman AM, Snyder SH (2001) Identification and characterization of a novel inositol hexakisphosphate kinase. J Biol Chem 276(42): 39179-39185.

10. Maag D, et al. (2011) Inositol polyphosphate multikinase is a physiologic PI3-kinase that activates Akt/PKB. Proc Natl Acad Sci USA 108(4):1391-1396.

11. Xu R, et al. (2013) Inositol polyphosphate multikinase is a coactivator of p53-mediated transcription and cell death. Sci Signal 6(269):ra22.

12. Kim S, et al. (2011) Amino acid signaling to mTOR mediated by inositol polyphosphate multikinase. Cell Metab 13(2):215-221.

13. Thiery JP, Acloque $H$, Huang RY, Nieto MA (2009) Epithelial-mesenchymal transitions in development and disease. Cell 139(5):871-890.

14. Cavallaro U, Dejana E (2011) Adhesion molecule signalling: Not always a sticky business. Nat Rev Mol Cell Biol 12(3):189-197.

15. Mitra SK, Hanson DA, Schlaepfer DD (2005) Focal adhesion kinase: in command and control of cell motility. Nat Rev Mol Cell Biol 6(1):56-68.

16. Carretero J, et al. (2010) Integrative genomic and proteomic analyses identify targets for Lkb1-deficient metastatic lung tumors. Cancer Cell 17(6):547-559.

17. Eneling $K$, et al. (2012) Salt-inducible kinase 1 regulates E-cadherin expression and intercellular junction stability. FASEB J 26(8):3230-3239.

18. Wang G, et al. (2008) Promoter-hypermethylation associated defective expression of E-cadherin in primary non-small cell lung cancer. Lung Cancer 62(2):162-172.

19. Roy BC, et al. (2010) Involvement of LKB1 in epithelial-mesenchymal transition (EMT) of human lung cancer cells. Lung Cancer 70(2):136-145.

20. Alessi DR, Sakamoto K, Bayascas JR (2006) LKB1-dependent signaling pathways. Annu Rev Biochem 75:137-163.

21. Lizcano JM, et al. (2004) LKB1 is a master kinase that activates 13 kinases of the AMPK subfamily, including MARK/PAR-1. EMBO J 23(4):833-843.

22. Jansen M, Ten Klooster JP, Offerhaus GJ, Clevers H (2009) LKB1 and AMPK family signaling: The intimate link between cell polarity and energy metabolism. Physiol Rev 89(3):777-798.

23. Baas AF, et al. (2003) Activation of the tumour suppressor kinase LKB1 by the STE20 like pseudokinase STRAD. EMBO J 22(12):3062-3072.
Inositol Profiling. Radiolabeling with $\left[{ }^{3} \mathrm{H}\right]$ inostiol and inositol phosphate detection was done as previously described (5).

Statistical Analysis. Results are presented as the mean and SE of at least three independent experiments. Statistical significance was calculated by Student's $t$ test $\left(* * P<0.05,{ }^{*} P<0.01\right)$, except for the metastasis percentage data, the statistical significance of which was calculated by a two-tailed Z-test for population proportions.

Cell migration/invasion, collagen gel invasion assay, centrifugation adhesion assay, mice xenograft and orthotopic model, and sources of antibodies are described in detail in SI Materials and Methods.

ACKNOWLEDGMENTS. We thank members of the S.H.S. laboratory for reagents and discussions. This work was supported by Public Health Service Grant DA-000266 (to S.H.S.). M.M.G. is supported by the National Institutes of Health Medical Scientist Training Program Award T32 GM007309.

24. Song P, et al. (2008) Protein kinase Czeta-dependent LKB1 serine 428 phosphorylation increases LKB1 nucleus export and apoptosis in endothelial cells. J Biol Chem 283(18) 12446-12455.

25. Koldobskiy MA, et al. (2010) p53-mediated apoptosis requires inositol hexakisphosphate kinase-2. Proc Natl Acad Sci USA 107(49):20947-20951.

26. Rajput A, et al. (2008) Characterization of HCT116 human colon cancer cells in an orthotopic model. J Surg Res 147(2):276-281.

27. Luo HR, et al. (2001) GRAB: A physiologic guanine nucleotide exchange factor for Rab3A, which interacts with inositol hexakisphosphate kinase. Neuron 31(3):439-451.

28. Brehm MA, Wundenberg T, Williams J, Mayr GW, Shears SB (2013) A non-catalytic role for inositol 1,3,4,5,6-pentakisphosphate 2-kinase in the synthesis of ribosomal RNA. J Cell Sci 126(Pt 2):437-444.

29. Wang H, DeRose EF, London RE, Shears SB (2014) IP6K structure and the molecula determinants of catalytic specificity in an inositol phosphate kinase family. Nat Commun 5:4178.

30. Moser AR, Pitot HC, Dove WF (1990) A dominant mutation that predisposes to multiple intestinal neoplasia in the mouse. Science 247(4940):322-324.

31. Zhang QC, et al. (2012) Structure-based prediction of protein-protein interactions on a genome-wide scale. Nature 490(7421):556-560.

32. Bang S, Chen Y, Ahima RS, Kim SF (2014) Convergence of IPMK and LKB1-AMPK signaling pathways on metformin action. Mol Endocrinol 28(7):1186-1193.

33. Kline ER, Shupe J, Gilbert-Ross M, Zhou W, Marcus Al (2013) LKB1 represses focal adhesion kinase (FAK) signaling via a FAK-LKB1 complex to regulate FAK site maturation and directional persistence. J Biol Chem 288(24):17663-17674.

34. Goodwin JM, et al. (2014) An AMPK-independent signaling pathway downstream of the LKB1 tumor suppressor controls Snail1 and metastatic potential. Mol Cell 55(3): $436-450$

35. Okon IS, Coughlan KA, Zou MH (2014) Liver kinase B1 expression promotes phosphatase activity and abrogation of receptor tyrosine kinase phosphorylation in human cancer cells. J Biol Chem 289(3):1639-1648.

36. Koivunen JP, et al. (2008) Mutations in the LKB1 tumour suppressor are frequently detected in tumours from Caucasian but not Asian lung cancer patients. Br J Cance 99(2):245-252.

37. Morrison BH, Bauer JA, Kalvakolanu DV, Lindner DJ (2001) Inositol hexakisphosphate kinase 2 mediates growth suppressive and apoptotic effects of interferon-beta in ovarian carcinoma cells. J Biol Chem 276(27):24965-24970.

38. Aoki M, Sobek V, Maslyar DJ, Hecht A, Vogt PK (2002) Oncogenic transformation by beta-catenin: Deletion analysis and characterization of selected target genes. Onco gene 21(46):6983-6991.

39. Roca $\mathrm{H}$, et al. (2013) Transcription factors OVOL1 and OVOL2 induce the mesenchymal to epithelial transition in human cancer. PLOS ONE 8(10):e76773.

40. Nilsson J, et al. (2010) Nuclear Janus-activated kinase 2/nuclear factor 1-C2 suppresses tumorigenesis and epithelial-to-mesenchymal transition by repressing Forkhead box F1. Cancer Res 70(5):2020-2029.

41. Barcellos-Hoff MH, Cucinotta FA (2014) New tricks for an old fox: Impact of TGF $\beta$ on the DNA damage response and genomic stability. Sci Signal 7(341):re5.

42. Ewan KB, et al. (2002) Transforming growth factor-beta1 mediates cellular response to DNA damage in situ. Cancer Res 62(20):5627-5631.

43. Wang H, et al. (2013) Epithelial-mesenchymal transition (EMT) induced by TNF- $\alpha$ re quires AKT/GSK-3 $\beta$-mediated stabilization of snail in colorectal cancer. PLOS ONE 8(2): e56664. 